\title{
A CLASS OF OVERDETERMINED SYSTEMS DEFINED BY TABLEAUX: INVOLUTIVENESS AND CAUCHY PROBLEM
}

\author{
EMILIO MUSSO AND LORENZO NICOLODI
}

\begin{abstract}
This article addresses the question of involutiveness and discusses the initial value problem for a class of overdetermined systems of partial differential equations which arise in the theory of integrable systems and are defined by tableaux.
\end{abstract}

\section{INTRODUCTION}

The present paper is concerned with the overdetermined system of first order nonlinear partial differential equations of type

$$
Q_{\alpha i}^{a} \partial_{x^{j}} F^{\alpha}-Q_{\alpha j}^{a} \partial_{x^{i}} F^{\alpha}=\Phi_{i j}^{a} \quad(1 \leq i<j \leq n),
$$

where $Q_{\alpha i}^{a}(a=1, \ldots r ; \alpha=1, \ldots, s)$ are constant coefficients and the nonhomogeneous terms $\Phi_{i j}^{a}$ are analytic functions depending on both the independent and dependent variables. The interest in this system originates from the observation that many soliton equations of mathematical physics and integrable systems occurring in submanifold geometry can be written in the form (1.1). Example include the equation of harmonic maps from Euclidean or Minkowski 2-space to a Lie group or a symmetric space (the principal chiral model in particle physics) 12, 14, 16; the Ward equation (or the modified $2+1$ chiral model) 4, 5]; the curved flat system of Ferus and Pedit [6, 7]; and the $G / G_{0}$-system of Terng associated to a rank $n$ symmetric space $G / G_{0}$ [13, 15, to name just a few.

One purpose of this paper is to address the question of involutiveness for the system (1.1). Roughly speaking, this problem amounts to finding sufficient conditions under which the system can be solved by repeated use of equations of CauchyKowalewski type. In the language of exterior differential systems (EDS), an involutive system is one that satisfies the hypotheses of the Cartan-Kähler theorem. R. Bryant, using the theory of EDS, proved that the $G / G_{0}$-system and the curved flat system are involutive (see Terng and Wang 15]). This result was taken up and extended in [10] where the two previous systems were identified as examples of a class of involutive systems defined by algebraic tableaux over Lie algebras. In this paper, we determine sufficient conditions (more general than the involutiveness condition) on the constant coefficients and the non-homogeneous terms of (1.1) in order to guarantee that the system becomes involutive after a sufficiently large number of successive prolongations. A second purpose of the work is to discuss the Cauchy

Date: February 28, 2006.

2000 Mathematics Subject Classification. Primary 58A15, 58A17; Secondary 35Q58, 53C42, $58 \mathrm{~F} 07$.

Authors partially supported by the MIUR projects Proprietà geometriche delle varietà reali e complesse and Metriche riemanniane e varietà differenziali, and the GNSAGA of INDAM. 
problem for the system (1.1), namely formulate appropriate initial conditions and prove a (local) existence and uniqueness theorem for the K-regular analytic solutions satisfying given initial conditions. As concerns the approach to the problem, these results are in line with those obtained by the authors in [11]. (For another view on this topic see also 8 .)

We use methods by which the above analytic questions may be reduced to algebraic questions concerning the tableau generated by the matrices $Q_{\alpha}=\left(Q_{\alpha i}^{a}\right)$ and the associated Spencer complex. This reduction essentially proceeds in three steps: (1) replacing the consideration of the system (1.1) by that of the differential ideal $\mathfrak{I}$ on $M=\mathbb{R}^{n} \oplus \mathbb{R}^{s}$ generated by the 2 -forms

$$
\Theta^{a}=Q_{\alpha i}^{a} d y^{\alpha} \wedge d x^{i}-\Phi_{j i}^{a} d x^{j} \wedge d x^{i} \quad(a=1, \ldots, r)
$$

and with independence (transversality) condition $\Omega=d x^{1} \wedge \cdots \wedge d x^{n} \neq 0$; (2) replacing the consideration of $(M, \mathfrak{I})$ by that of its first prolongation $\left(M_{(1)}, \mathfrak{I}_{(1)}\right)$, that is, the restriction to the space of $n$-dimensional integral elements of $\mathfrak{I}$ of the canonical Pfaffian system on the Grassmann bundle $G_{n}(T M)$ of tangent $n$-planes of $M$; (3) replacing the consideration of this Pfaffian system and its higher prolongations $\left(M_{(h)}, \mathfrak{I}_{(h)}\right)$ by that of the tableau $\left(Q_{\alpha i}^{a}\right)$, its prolongations, and the harmonic decomposition of the Spencer complex attached to it.

In Section 2 we review the needed results about the algebraic theory of tableaux, we introduce the Spencer complex associated to a tableau, and describe the Guillemin normal form for an involutive tableau.

In Section 3] we carry out the steps of the above list and present the results concerning the involutiveness of (1.1). We prove that if the tableau $\left(Q_{\alpha i}^{a}\right)$ is 2acyclic and the non-homogeneous term $\Phi$ satisfies reasonable regularity conditions (satisfied by all the cited examples), then the prolongation tower

$$
\cdots \stackrel{\pi_{h+1}}{\longrightarrow}\left(M_{(h)}, \mathfrak{I}_{(h)}, \Omega\right) \stackrel{\pi_{h}}{\longrightarrow}\left(M_{(h-1)}, \mathfrak{I}_{(h-1)}, \Omega\right) \stackrel{\pi_{h-1}}{\longrightarrow} \cdots \stackrel{\pi_{2}}{\longrightarrow}\left(M_{(1)}, \mathfrak{I}_{(1)}, \Omega\right)
$$

is indeed a Frobenius tower; here we use the terminology introduced by Bryant and Griffiths in the foundational paper [3] about the characteristic cohomology of differential systems (see Section 3 for the definition). Next, we prove that if the $k$-th prolongation of the tableau $\left(Q_{\alpha i}^{a}\right)$ is involutive, then the $k$-th prolongation $\mathfrak{I}_{(k)}$

of $\mathfrak{I}$ is also involutive and has the same characters of the involutive prolongation of the tableau.

In Section 4 we show that the examples listed above all share the common formalism (1.1) and fit into the general scheme.

In the final section, we discuss the Cauchy problem for the system (1.1); the existence and uniqueness of K-regular analytic solutions for suitable analytic Cauchy data is proved. The basic reference on exterior differential systems has been [2] and our notation and terminology are consistent with that reference. Summation convention over repeated indices will be adopted throughout the paper.

\section{Involutive tableaux And the SPencer COMPleX}

In this section we briefly review the material and the results we need in the algebraic theory of tableaux, referring for details to the book by Bryant, et al. 2]. 
2.1. Definitions and basic properties. Let $\mathfrak{a}$ and $\mathfrak{b}$ be (real or complex) finite dimensional vector spaces. A tableau $\mathbf{A}$ is a linear subspace of $\operatorname{Hom}(\mathfrak{a}, \mathfrak{b})$.

An $h$-dimensional subspace $\mathfrak{a}_{h} \subset \mathfrak{a}$ is called generic with respect to $\mathbf{A}$ if the dimension of $\operatorname{Ker}\left(\mathbf{A}, \mathfrak{a}_{h}\right):=\left\{Q \in \mathbf{A} \mid Q_{\left.\right|_{\mathfrak{a}_{h}}}=0\right\}$ is a minimum, i.e.,

$$
\operatorname{dim} \operatorname{Ker}\left(\mathbf{A}, \mathfrak{a}_{h}\right)=\min \left\{\operatorname{dim} \operatorname{Ker}\left(\mathbf{A}, \tilde{\mathfrak{a}}_{h}\right): \tilde{\mathfrak{a}}_{h} \in G_{h}(\mathfrak{a})\right\},
$$

where $G_{h}(\mathfrak{a})$ denotes the Grassmannian of $h$-dimensional subspaces of $\mathfrak{a}$. Similarly, a flag $(0) \subset \mathfrak{a}_{1} \subset \cdots \subset \mathfrak{a}_{n}=\mathfrak{a}$ of $\mathfrak{a}$ is said generic if $\mathfrak{a}_{h}$ is generic for all $h=1, \ldots, n$. The set of generic flags is an open and dense subset of the flag manifold $\mathcal{F}(\mathfrak{a})$.

The characters of $\mathbf{A}$ are the non-negative integers $s_{j}(\mathbf{A}), j=1, \ldots, n$, defined inductively by

$$
s_{1}(\mathbf{A})+\cdots+s_{j}(\mathbf{A})=\operatorname{codim} \operatorname{Ker}\left(\mathbf{A}, \mathfrak{a}_{j}\right), \quad j=1, \ldots, n,
$$

where $(0) \subset \mathfrak{a}_{1} \subset \cdots \subset \mathfrak{a}_{n}=\mathfrak{a}$ is a generic flag. From the definition it is clear that

$$
\operatorname{dim} \mathfrak{b} \geq s_{1} \geq s_{2} \geq \cdots \geq s_{n}, \quad \operatorname{dim} \mathbf{A}=s_{1}+\cdots+s_{n} .
$$

If $s_{\nu} \neq 0$, but $s_{\nu+1}=0$, we say that $\mathbf{A}$ has principal character $s_{\nu}$ and call $\nu$ the Cartan integer of $\mathbf{A}$.

The first prolongation of $\mathbf{A}$ is the subspace $\mathbf{A}^{(1)} \subset \mathbf{A} \otimes \mathfrak{a}^{*} \simeq \operatorname{Hom}(\mathfrak{a}, \mathbf{A})$ of all linear transformations $Q: \mathfrak{a} \rightarrow \mathbf{A}$ such that

$$
Q(X)(Y)=Q(Y)(X), \quad \text { for all } X, Y \in \mathfrak{a} .
$$

Notice that $\mathbf{A}^{(1)}$ is itself a tableau and that $\mathbf{A}^{(1)}=\left(\mathbf{A} \otimes \mathfrak{a}^{*}\right) \cap\left(\mathfrak{b} \otimes S^{2}\left(\mathfrak{a}^{*}\right)\right)$. (By $S^{h}\left(\mathfrak{a}^{*}\right)$ we denote the symmetric $h$-fold tensor product of $\mathfrak{a}^{*}$.) The $h$-th prolongation of $\mathbf{A}$ is defined inductively by setting $\mathbf{A}^{(h)}=\mathbf{A}^{(h-1)}{ }^{(1)}$, for $h \geq 1$, where $\mathbf{A}^{(0)}=\mathbf{A}$ and by convention $\mathbf{A}^{(-1)}=\mathfrak{b}$. The prolongation $\mathbf{A}^{(h)}$ is identified with

$$
\mathbf{A}^{(h)}=\left(\mathbf{A} \otimes S^{h}\left(\mathfrak{a}^{*}\right)\right) \cap\left(\mathfrak{b} \otimes S^{h+1}\left(\mathfrak{a}^{*}\right)\right)
$$

and an element $Q_{(h)} \in \mathfrak{b} \otimes S^{h+1}\left(\mathfrak{a}^{*}\right)$ belongs to $\mathbf{A}^{(h)}$ if and only if $i(X) Q_{(h)} \in$ $\mathbf{A}^{(h-1)}$, for all $X \in \mathfrak{a}$. The direct sum

$$
\mathbf{A}^{[\infty]}=\bigoplus_{h \geq 0} \mathbf{A}^{(h)},
$$

is called total prolongation of $\mathbf{A}$. The total prolongation has a natural filtration

$$
\mathbf{A}=\mathbf{A}^{[0]} \subset \mathbf{A}^{[1]} \subset \mathbf{A}^{[2]} \subset \cdots \subset \mathbf{A}^{[p]} \subset \cdots \subset \mathbf{A}^{(\infty)},
$$

where $\mathbf{A}^{[p]}=\bigoplus_{0 \leq h \leq p} \mathbf{A}^{(h)}$.

One can establish the following result (cf. 2])

$$
\operatorname{dim} \mathbf{A}^{(1)} \leq s_{1}+2 s_{2}+\cdots+n s_{n} .
$$

A is said involutive (or in involution) if equality holds in the above inequality.

Also, for any tableau $\mathbf{A}$, there exists an integer $h_{0}$ such that $\mathbf{A}^{(h)}$ is involutive, for all $h \geq h_{0}$. We call the smallest integer $k$ with this property the involutive index and the corresponding prolongation the involutive prolongation of the tableau. The involutive characters of $\mathbf{A}$ are the characters of the involutive prolongation $\mathbf{A}^{(k)}$. Accordingly, we have the notion of involutive principal character and involutive Cartan integer of a tableau. 
Another basic property is that every prolongation of an involutive tableau is involutive. In particular, if $\mathbf{A}$ is involutive, the characters of $\mathbf{A}$ and $\mathbf{A}^{(1)}$ are related by

$$
s_{j}^{(1)}:=s_{j}\left(\mathbf{A}^{(1)}\right)=s_{n}(\mathbf{A})+\cdots+s_{j}(\mathbf{A}) \quad(j=1, \ldots, n) .
$$

This implies that the principal character and the Cartan integer are invariant under prolongation of an involutive tableau.

2.2. The Spencer complex. With the previous notation, let

$$
\delta^{q, p}: \mathfrak{b} \otimes S^{q}\left(\mathfrak{a}^{*}\right) \otimes \Lambda^{p}\left(\mathfrak{a}^{*}\right) \rightarrow \mathfrak{b} \otimes S^{q-1}\left(\mathfrak{a}^{*}\right) \otimes \Lambda^{p+1}\left(\mathfrak{a}^{*}\right)
$$

be the operator given by

$$
\delta^{q, p} \phi\left(X_{1}, \ldots, X_{p+1}\right):=\sum_{k}(-1)^{k+1} i\left(X_{k}\right) \phi\left(X_{1}, \ldots, \hat{X}_{k}, \ldots, X_{p+1}\right) .
$$

If $q=0$ we set $\delta^{0, p}=0$, for $p \geq 0$. From the definition, we have that $\delta \circ \delta=0$. Also, the sequence of the corresponding bi-graded complex is exact except when $q=0$ and $p=0$.

Let $\mathbf{A} \subset \operatorname{Hom}(\mathfrak{a}, \mathfrak{b})$ be a tableau with prolongations $\mathbf{A}^{(h)}, h \geq 0$. Consider the sequence of spaces

$$
C^{q, p}(\mathbf{A}):=\mathbf{A}^{(q-1)} \otimes \Lambda^{p}\left(\mathfrak{a}^{*}\right),
$$

for integers $q \geq 0$ and $0 \leq p \leq n$. Since $\mathbf{A}^{(q-1)} \subset \mathfrak{b} \otimes S^{q}\left(\mathfrak{a}^{*}\right)$, the space $C^{q, p}(\mathbf{A})$ is a subspace of $\mathfrak{b} \otimes S^{q}\left(\mathfrak{a}^{*}\right) \otimes \Lambda^{p}\left(\mathfrak{a}^{*}\right)$. Now the sequence of subspaces $C^{q, p}(\mathbf{A})$ is stable under $\delta$, that is, $\delta C^{q, p}(\mathbf{A}) \subset C^{q-1, p+1}(\mathbf{A})$. In fact, for any $\phi \in C^{q, p}(\mathbf{A})$, each summand in the r.h.s. of (2.2) lies in $\mathbf{A}^{(q-2)}$ by the defining property of prolongations. We still have $\delta \circ \delta=0$ (for $q \geq 1$ and $p=1, \ldots, n$ ), but the sequence

$$
C^{q+1, p-1}(\mathbf{A}) \stackrel{\delta^{q+1, p-1}}{\longrightarrow} C^{q, p}(\mathbf{A}) \stackrel{\delta^{q, p}}{\longrightarrow} C^{q-1, p+1}(\mathbf{A})
$$

is no longer exact for all $p$ and $q$. The associated cohomology groups

$$
H^{q, p}(\mathbf{A}):=Z^{q, p}(\mathbf{A}) / B^{q, p}(\mathbf{A})
$$

are called the Spencer groups of $\mathbf{A}$, where $B^{q, p}(\mathbf{A})=\operatorname{Im}\left(\delta^{q+1, p-1}\right)$ and $Z^{q, p}(\mathbf{A})=$ $\operatorname{Ker}\left(\delta^{q, p}\right)$. Notice that $Z^{0, p}(\mathbf{A})=\mathfrak{b} \otimes \Lambda^{p}\left(\mathfrak{a}^{*}\right)$ and $Z^{q, 1}(\mathbf{A})=\mathbf{A}^{(q)}$, for all $q \geq 1$, $p \geq 0$.

Remark 2.1. A decomposable element of $C^{q, p}(\mathbf{A}), q \geq 1$, is of the form $\mathcal{Q}=$ $Q \otimes\left(\alpha^{i_{1}} \wedge \cdots \wedge \alpha^{i_{p}}\right)$, where $Q \in \mathbf{A}^{(q-1)} \subset \operatorname{Hom}\left(\mathfrak{a}, \mathbf{A}^{(q-2)}\right)$. If $\left(\bar{Q}_{1}^{(q-2)}, \ldots, \bar{Q}_{r_{q-2}}^{(q-2)}\right)$ is a basis of $\mathbf{A}^{(q-2)}$, then $Q=Q_{i}^{a} \bar{Q}_{a}^{(q-2)} \otimes \eta^{i}$ and

$$
Q \otimes\left(\alpha^{i_{1}} \wedge \cdots \wedge \alpha^{i_{p}}\right)=Q_{i}^{a}\left(\bar{Q}_{a}^{(q-2)} \otimes \eta^{i}\right) \otimes\left(\alpha^{i_{1}} \wedge \cdots \wedge \alpha^{i_{p}}\right) .
$$

Then $\delta(\mathcal{Q})$ is the element of $C^{q-1, p+1}(\mathbf{A})$ defined by

$$
\delta(\mathcal{Q})=Q_{i}^{a} \bar{Q}_{a}^{(q-2)} \otimes\left(\eta^{i} \wedge \alpha^{i_{1}} \wedge \cdots \wedge \alpha^{i_{p}}\right) .
$$

A significant result in the subject is that the vanishing of the $H^{q, p}$ is equivalent to involutiveness.

Theorem 2.2. A tableau $\mathbf{A}$ is involutive if and only if $H^{q, p}(\mathbf{A})$ is zero, for all $q \geq 1$ and $p \geq 0$. 
If $H^{q, 2}(\mathbf{A})=(0)$, for all $q \geq 1$, the tableau $\mathbf{A}$ is called 2-acyclic.

Suppose that $\mathfrak{a}$ and $\mathfrak{b}$ are endowed with (positive definite) scalar products, and consider the scalar product induced on $C^{q, p}(\mathbf{A})=\mathbf{A}^{(p-1)} \otimes \Lambda^{p}\left(\mathfrak{a}^{*}\right)$. With respect to this scalar product, let

$$
\delta^{*}: C^{q-1, p+1}(\mathbf{A}) \rightarrow C^{q, p}(\mathbf{A})
$$

be the co-differential defined by $\left\langle\delta^{*}(\zeta), \rho\right\rangle=\langle\zeta, \delta(\rho)\rangle$, for $\zeta \in C^{q-1, p+1}(\mathbf{A})$ and $\rho \in C^{q, p}(\mathbf{A})$. This yields a harmonic decomposition of the Spencer complex:

$$
C^{q, p}(\mathbf{A})=B^{q, p}(\mathbf{A}) \oplus \mathcal{H}^{q, p}(\mathbf{A}) \oplus B_{q, p}(\mathbf{A})
$$

where $B^{q, p}(\mathbf{A})=\delta\left(C^{q+1, p-1}(\mathbf{A})\right), B_{q, p}(\mathbf{A})=\delta^{*}\left(C^{q-1, p+1}(\mathbf{A})\right)$, and $\mathcal{H}^{q, p}(\mathbf{A})=$ $\operatorname{Ker}(\delta) \cap \operatorname{Ker}\left(\delta^{*}\right)$. From the definition, it follows that:

- the application $\zeta \in \mathcal{H}^{q, p}(\mathbf{A}) \rightarrow[\zeta] \in H^{q, p}(\mathbf{A})$ is an isomorphism;

- $\operatorname{Ker}(\delta)=Z^{q, p}(\mathbf{A})=B^{q, p}(\mathbf{A}) \oplus \mathcal{H}^{q, p}(\mathbf{A})$;

- $\operatorname{Ker}\left(\delta^{*}\right)=Z_{q, p}(\mathbf{A})=\mathcal{H}^{q, p}(\mathbf{A}) \oplus B_{q, p}(\mathbf{A})$;

- $\mathcal{H}^{q, p}(\mathbf{A})=Z^{q, p}(\mathbf{A}) \cap Z_{q, p}(\mathbf{A})$.

Finally, observe that the restriction of the Spencer differential to $B_{q, p}(\mathbf{A})$ is an isomorphism onto $B^{q-1, p+1}(\mathbf{A})$. The inverse of $\delta_{\left.\right|_{B_{q}, p}(\mathbf{A})}$ will be denoted by

$$
\sigma_{q, p}: B^{q-1, p+1}(\mathbf{A}) \rightarrow B_{q, p}(\mathbf{A}) .
$$

2.3. The Guillemin normal form for involutive tableaux. Let $\mathbf{A} \subset \operatorname{Hom}(\mathfrak{a}, \mathfrak{b})$ be a tableau. Let $\mathcal{A}=\left(A_{1}, \ldots, A_{n}\right)$ denote any basis of $\mathfrak{a},\left(\alpha^{1}, \ldots, \alpha^{n}\right)$ its dual basis, and $\mathcal{B}=\left(B_{1}, \ldots, B_{r}\right)$ any basis of $\mathfrak{b}$. Identify $\operatorname{Hom}(\mathfrak{a}, \mathfrak{b})$ with the space of $r \times n$ matrices $\mathbb{K}(r, n)$ and consider $\mathbf{A}$ as a subspace of $\mathbb{K}(r, n)$. Let $\pi=\left(\pi_{j}^{a}\right)$ be the tautological 1-form on $\operatorname{Hom}(\mathfrak{a}, \mathfrak{b})$ and let $\pi_{1}, \ldots, \pi_{n}$ denote its column vectors. Set $\pi_{j}=\left(\pi_{j}^{[\nu]}, \ldots, \pi_{j}^{[0]}\right)^{T}$, where $\pi_{j}^{[\nu]}=\left(\pi_{j}^{1}, \ldots, \pi_{j}^{s_{\nu}}\right)^{T}, \pi_{j}^{[\rho]}=\left(\pi_{j}^{s_{\nu}+\cdots+s_{\rho+1}+1}, \ldots\right.$, $\left.\pi_{j}^{s_{\nu}+\cdots+s_{\rho}}\right)^{T}$, for $\rho=\nu-1, \ldots, 1$, and $\pi_{j}^{[0]}=\left(\pi_{j}^{s_{\nu}+\cdots+s_{1}+1}, \ldots, \pi_{j}^{r}\right)^{T}$.

The following result is essentially due to Guillemin (cf. [2], 9]).

Proposition 2.3. If $\mathbf{A} \subset \operatorname{Hom}(\mathfrak{a}, \mathfrak{b})$ is involutive, the bases $\mathcal{A}$ and $\mathcal{B}$ can be chosen so that $\left.\pi_{i}^{[0]}\right|_{\mathbf{A}}=0$, for $i \geq 1,\left.\pi_{i}^{[\rho]}\right|_{\mathbf{A}} \in \operatorname{span}\left(\left.\pi_{1}^{1}\right|_{\mathbf{A}}, \ldots,\left.\pi_{1}^{s_{1}}\right|_{\mathbf{A}}, \ldots,\left.\pi_{\rho}^{1}\right|_{\mathbf{A}}, \ldots\right.$, $\left.\left.\pi_{\rho}^{s_{\rho}}\right|_{\mathbf{A}}\right)$, for $\rho=1, \ldots, \nu$ and $i \geq \rho$. In particular, $\left(\left.\pi_{1}^{1}\right|_{\mathbf{A}}, \ldots,\left.\pi_{1}^{s_{1}}\right|_{\mathbf{A}},\left.\pi_{2}^{1}\right|_{\mathbf{A}}, \ldots,\left.\pi_{2}^{s_{2}}\right|_{\mathbf{A}}\right.$, $\left.\ldots,\left.\pi_{\nu}^{1}\right|_{\mathbf{A}}, \ldots,\left.\pi_{\nu}^{s_{\nu}}\right|_{\mathbf{A}}\right)$ is a basis of $\mathbf{A}^{*}$ and the elements of its dual basis

$$
\mathcal{Q}=\left(Q_{[1], 1}, \ldots, Q_{[1], s_{1}}, Q_{[2], 1}, \ldots, Q_{[2], s_{2}}, \ldots, Q_{[\nu], 1}, \ldots, Q_{[\nu], s_{\nu}}\right)
$$

can be expressed by

$$
\begin{gathered}
Q_{[j], a}=B_{a} \otimes \alpha^{j}+\sum_{h=j+1}^{\nu}\left(\sum_{b=s_{h}+1}^{s_{h+1}} Q_{[j], a, h}^{b} B_{b} \otimes \alpha^{h}\right)+ \\
+\sum_{h=\nu+1}^{n}\left(\sum_{b=1}^{s_{\nu}} Q_{[j], a, h}^{b} B_{b} \otimes \alpha^{h}\right),
\end{gathered}
$$

for all $j=1, \ldots, \nu ; a=1, \ldots, s_{j}$.

We say that $\mathcal{Q}$ is a normal basis of the involutive tableau $\mathbf{A}$ and that the expression (2.3) is the Guillemin normal form for the elements of $\mathcal{Q}$ with respect to the basis $\mathcal{A}$ and $\mathcal{B}$.

Remark 2.4. Notice that the basis $\left(A_{1}, \ldots, A_{n}\right)$ of $\mathfrak{a}$ is automatically generic with respect to the tableau $\mathbf{A}$, that is, the corresponding flag: $(0) \subset \mathfrak{a}_{1}=\operatorname{span}\left(A_{1}\right) \subset$ $\mathfrak{a}_{2}=\operatorname{span}\left(A_{1}, A_{2}\right) \subset \cdots \subset \mathfrak{a}=\operatorname{span}\left(A_{1}, \ldots, A_{n}\right)$ is generic. 


\section{DifFERENTIAL IDEALS ASSOCIATED WITH TABLEAUX}

Notation 3.1. We start by recalling some terminology and notation. An exterior differential system (EDS) is a pair $(M, \mathfrak{I})$ consisting of a smooth manifold $M$ and a differential ideal $\mathfrak{I}$, i.e., a homogeneous, differentially closed ideal $\mathfrak{I} \subseteq \Omega^{*}(M)$ in the algebra of smooth differential forms on $M$. A Pfaffian differential system is an EDS which is differentially generated by 1 -forms. It is given by the sections of a smooth subbundle $I \subset T^{*} M$ of the cotangent bundle; the space of section of this subbundle, and sometimes the subbundle itself, is also referred to as a Pfaffian system. We use the notation $\{\alpha, \beta, \ldots\}$ for the (two-sided) algebraic ideal generated by forms $\alpha, \beta, \ldots$, and $\{I\}$ for the algebraic ideal generated by the sections of a Pfaffian system $I \subseteq T^{*} M$.

With reference to the system (1.1), let $\mathfrak{a}=\mathbb{R}^{n}, \mathfrak{b}=\mathbb{R}^{s}$ and denote by $\mathbf{A} \subset$ $\operatorname{Hom}(\mathfrak{a}, \mathfrak{b})$ the tableau generated by the matrices $Q_{\alpha}=\left(Q_{\alpha i}^{a}\right) \in \mathbb{K}(r, n), \alpha=$ $1, \ldots, s$. Assume that the $Q_{\alpha}$ be linearly independent so that $\mathfrak{b}$ can be identified with $\mathbf{A}$, and let $q_{(0)}^{\alpha}$ denote the coordinates with respect to $Q_{\alpha}$. Let $\mathfrak{I} \subset \Omega^{*}(M)$ be the differential ideal on $M=\mathfrak{a} \oplus \mathbf{A}$ generated by the 2 -forms

$$
\Theta^{a}=Q_{\alpha j}^{a} d q_{(0)}^{\alpha} \wedge d x^{j}-\Phi_{i j}^{a} d x^{i} \wedge d x^{j} \quad(a=1, \ldots, r),
$$

where $\Phi_{i j}^{a}$ are analytic maps of the $x^{i}$ and the $q_{(0)}^{\alpha}$, and $\Omega=d x^{1} \wedge \cdots \wedge d x^{n} \neq 0$ gives the independence (transversality) condition. Locally, the integral manifolds of $(\mathfrak{I}, \Omega)$ can be expressed as graphs of solutions to (1.1). Further, we assume that $\Phi(x, q)=\left(\Phi_{i j}^{a}\right): M \rightarrow C^{0,2}(\mathbf{A})$ takes values in $B^{0,2}(\mathbf{A})$ and satisfies

$$
\left.\sum_{(1,2,3)} \Phi_{*}\right|_{(A, Q)}\left(A_{1}+Q_{(1)}\left(A_{1}\right)\right)\left(A_{2}, A_{3}\right)=0
$$

(sum over cyclic permutations), for $(A, Q) \in M, A_{1}, A_{2}, A_{3} \in \mathfrak{a}$, and $Q_{(1)} \in \mathbf{A}^{(1)}$, where $\Phi_{*}: T_{(A, Q)} M \simeq M \rightarrow T_{\Phi(A, Q)} B^{0,2}(\mathbf{A}) \simeq B^{0,2}(\mathbf{A})$ denotes the differential of $\Phi$ at $(A, Q)$. (In the next section, we will see that the examples cited in the introduction all satisfy these conditions.)

Definition 3.2. We call $(M, \mathfrak{I}, \Omega)$ the differential system associated with the tableau A and the non-homogeneous term $\Phi$.

Next, consider a harmonic decomposition of the Spencer complex, and in particular the decomposition

$$
C^{1,1}(\mathbf{A})=B^{1,1}(\mathbf{A}) \oplus \mathcal{H}^{1,1}(\mathbf{A}) \oplus B_{1,1}(\mathbf{A}) .
$$

Let $S_{(1)}: M \rightarrow B_{1,1}(\mathbf{A})$ be the analytic mapping defined by $\delta^{1,1}\left(S_{(1)}\right)=\Phi$. Then

$$
\Theta^{a}=Q_{\alpha j}^{a}\left(d q_{(0)}^{\alpha}+S_{(1)}^{\alpha} d x^{i}\right) \wedge d x^{j},
$$

from which follows that the $n$-dimensional integral elements of the differential system $(\mathfrak{I}, \Omega)$ are generated by vectors of the form

$$
v_{i}=\partial_{x^{i}}-\left(S_{(1)_{i}^{\alpha}}^{\alpha}+Q_{(1)_{i}}^{\alpha}\right) \partial_{q_{(0)}^{\alpha}} \quad(i=1, \ldots, n),
$$

where $Q_{(1)}=\left(Q_{(1)}^{\alpha}\right) \in \mathbf{A}^{(1)}$. Therefore, the space $V_{n}(\mathfrak{I}, \Omega)$ of $n$-dimensional integral elements can be identified with $M_{(1)}=\mathfrak{a} \oplus \mathbf{A}^{[1]}$ and the restriction to $V_{n}(\mathfrak{I}, \Omega)$ of the canonical contact Pfaffian system on the Grassmann bundle $G_{n}(T M)$ (i.e., the 
first prolongation of $\mathfrak{I}$ ) coincides with the Pfaffian system given by the subbundle $I_{(1)} \subset T^{*}\left(M_{(1)}\right)$, whose sections are the components of the 1-form

$$
\beta_{(0)}:=d Q_{(0)}+\left(S_{(1)}+Q_{(1)}\right) \cdot d x
$$

so that locally $I_{(1)}=\operatorname{span}\left\{\beta_{(0)}\right\}$.

Definition 3.3. Let $\left(M_{(k)}, \mathfrak{I}_{(k)}, \Omega\right), k \geq 1$, be a sequence of smooth manifolds $M_{(k)}$ and Pfaffian systems $\mathfrak{I}_{(k)}$ with surjective submersions $\pi_{k}: M_{(k)} \rightarrow M_{(k-1)}$ such that $\pi_{k}^{*}\left(\mathfrak{I}_{(k-1)}\right) \subseteq \mathfrak{I}_{(k)}$. Let $I_{(k)} \subset T^{*} M_{(k)}$ be the subbundle whose sections are the 1 -forms in $\mathfrak{I}_{(k)}$. The above data define a Frobenius tower if $d I_{(k)} \equiv 0$ $\bmod \left\{I_{(k+1)}\right\}$ (cf. [3]).

We are now in the position to prove the following.

Theorem 3.4. Let $\mathbf{A} \subset \operatorname{Hom}(\mathfrak{a}, \mathfrak{b})$ be 2-acyclic and assume $\Phi: M \rightarrow B^{0,2}(\mathbf{A})$ is analytic and satisfies (3.1). Then the following statements hold true:

(1) $\left(M_{(1)}, \mathfrak{I}_{(1)}, \Omega\right)$ is a quasi-linear Pfaffian system with vanishing torsion;

(2) $\left(M_{(1)}, \mathfrak{I}_{(1)}, \Omega\right)$ admits a Frobenius tower

$$
\cdots \rightarrow\left(M_{(h)}, \mathfrak{I}_{(h)}, \Omega\right) \rightarrow\left(M_{(h-1)}, \mathfrak{I}_{(h-1)}, \Omega\right) \rightarrow \cdots \rightarrow\left(M_{(1)}, \mathfrak{I}_{(1)}, \Omega\right) ;
$$

(3) if $k$ is the least integer such that $\mathbf{A}^{(k)}$ is involutive, then $\left(M_{(k)}, \Im_{(k)}, \Omega\right)$ is involutive and its Cartan characters coincide with the characters of $\mathbf{A}^{(k)}$.

Proof. Let ${ }^{1}$

$$
\overline{\mathcal{D}} S_{(1)}:=\left(\partial_{x} S_{(1)}+\partial_{Q_{(0)}} S_{(1)}\left(S_{(1)}+Q_{(1)}\right)(d x)\right) \dot{\wedge} d x .
$$

Then $\Phi$ satisfies (3.1) if and only if $\delta^{1,2}\left(\overline{\mathcal{D}} S_{(1)}\right)=0$. Since $\mathbf{A}$ is 2-acyclic, there exists a unique analytic map $S_{(2)}: M_{(1)} \rightarrow B_{2,1}(\mathbf{A})$ such that $\delta^{2,1}\left(S_{(2)}\right)=-\overline{\mathcal{D}} S_{(1)}$. With this notation, the structure equations of $\left(\mathfrak{I}_{(1)}, \Omega\right)$ become

$$
d \beta_{(0)} \equiv-\left(d Q_{(1)}-S_{(2)}(d x)\right) \dot{\wedge} d x \quad \bmod \left\{I_{(1)}\right\} .
$$

This yields that $\left(\mathfrak{I}_{(1)}, \Omega\right)$ is a linear Pfaffian system with vanishing torsion, proving (1).

For result (2), we proceed by induction on $h$. For each integer $h \geq 1$, let $M_{(h)}:=$ $\mathfrak{a} \oplus \mathbf{A}^{[h]}$ and denote its elements by $\left(x, Q_{(0)}, \ldots, Q_{(h)}\right)$. Consider the analytic map $S_{(h+1)}: M_{(h)} \rightarrow B_{h+1,1}(\mathbf{A})$ defined inductively by

$$
\delta^{1,1}\left(S_{(1)}\right)=\Phi ; \quad \delta^{r, 1}\left(S_{(r)}\right)=-\overline{\mathcal{D}}\left(S_{(r-1)}\right) \quad(r=2, \ldots, h),
$$

where

$$
\overline{\mathcal{D}}\left(S_{(r)}\right)=\left(\left(\partial_{x} S_{(r)}+\sum_{s=0}^{r-1} \partial_{Q_{(s)}} S_{(r)}\left(S_{(s+1)}+Q_{(s+1)}\right)\right) \dot{\wedge} d x\right) \dot{\wedge} d x
$$

Consider the 1-forms

$$
\begin{gathered}
\beta_{(r)}=d Q_{(r)}-\left(S_{(r+1)}+Q_{(r+1)}\right)(d x) \quad(r=0, \ldots, h-1), \\
\pi_{(h)}=d Q_{(h)}-S_{(h+1)}(d x),
\end{gathered}
$$

and the filtration of subbundles locally given by

$$
I_{(1)}=\operatorname{span}\left\{\beta_{(0)}\right\} \subset \cdots \subset I_{(h)}=\operatorname{span}\left\{\beta_{(0)}, \cdots, \beta_{(h-1)}\right\} \subset T^{*} M_{(h)}
$$

\footnotetext{
${ }^{1}$ If $\phi$ and $\psi$ are matrices of real-valued differential forms, $\phi \dot{\wedge} \psi$ denotes the matrix multiplication where the entries are multiplied via wedge product.
} 
with independence condition $\Omega=d x^{1} \wedge \cdots \wedge d x^{n}$. A direct computation yields that the structure equations of the Pfaffian system $\left(\mathfrak{I}_{(h)}, \Omega\right)$ can be written in the form:

$$
\begin{aligned}
d \beta_{(0)} & \equiv-\beta_{(1)} \dot{\wedge} d x, \quad \bmod \left\{I_{(1)}\right\}, \\
d \beta_{(1)} & \equiv-\beta_{(2)} \dot{\wedge} d x, \quad \bmod \left\{I_{(2)}\right\}, \\
& \cdots \cdots \\
d \beta_{(h-2)} & \equiv-\beta_{(h-1)} \dot{\wedge} d x \quad \bmod \left\{I_{(h-1)}\right\}, \\
d \beta_{(h-1)} & \equiv-\pi_{(h)} \dot{\wedge} d x \quad \bmod \left\{I_{(h)}\right\} .
\end{aligned}
$$

This implies that the prolongation tower

$$
\cdots \rightarrow\left(M_{(h)}, \mathfrak{I}_{(h)}, \Omega\right) \rightarrow\left(M_{(h-1)}, \mathfrak{I}_{(h-1)}, \Omega\right) \rightarrow \cdots \rightarrow\left(M_{(1)}, \mathfrak{I}_{(1)}, \Omega\right)
$$

is indeed a Frobenius tower (cf. [3]). The general inductive step is clear.

As for (3), let $k$ be the least integer such that $\mathbf{A}^{(k)}$ is involutive and let $s_{1}^{(k)}, \ldots$, $s_{n}^{(k)}$ be the involutive characters. Consider the Pfaffian system $\left(M_{(k)}, \mathfrak{I}_{(k)}, \Omega\right)$. From the structure equations, we see that $\pi_{(h)}$ is the tableau matrix of the system. This means that the reduced tableau matrix is the tautological form of $\mathbf{A}^{(k)}$. Knowing that $\mathbf{A}^{(k)}$ is involutive and using the Cartan test for involution of linear Pfaffian systems, it follows that $\left(\mathfrak{I}_{(k)}, \Omega\right)$ is involutive, with Cartan characters $s_{1}^{(k)}, \ldots, s_{n}^{(k)}$.

Remark 3.5. Let $M_{(\infty)}:=\mathfrak{a} \oplus \mathbf{A}^{[\infty]}$ and let $F: M_{(\infty)} \rightarrow \mathbb{K}$ be a function such that $F=\pi_{h} \circ f$, where $\pi_{h}: M_{(\infty)} \rightarrow M_{(h)}$ is the projection and $f: M_{(h)} \rightarrow \mathbb{K}$ is a differentiable (resp., analytic, holomorphic) function. Then $F$ is said of class $C^{\infty}$ (resp., analytic, holomorphic). Let $\Omega^{*}\left(M_{(\infty)}\right)$ be the graded differential algebra $C^{\infty}\left(M_{(\infty)}, \mathbb{K}\right) \otimes_{\mathbb{K}}\left(\oplus_{p=0}^{\infty} \Lambda^{p}\left(M_{(\infty)}\right)\right)$ (the exterior differentiation is defined as in the finite-dimensional case) and let $\mathfrak{I}^{\infty} \subset \Omega^{*}\left(M_{(\infty)}\right)$ be the Frobenius ideal generated by $\left\{\beta_{(h)}\right\}_{h \in \mathbb{N}}$. The characterisic complex ${ }^{2}$ of the differential system $(M, \mathfrak{I}, \Omega)$ is the quotient $\bar{\Omega}=\Omega^{*}\left(M_{(\infty)}\right) / \mathfrak{I}^{\infty}$ endowed with the coboundary operator induced, passing to quotient, by the exterior differentiation of $\Omega^{*}\left(M_{(\infty)}\right)$. In our case $\bar{\Omega}=$ $C^{\infty}\left(M_{(\infty)}, \mathbb{K}\right) \otimes_{\mathbb{K}} \Lambda^{*}(\mathfrak{a})$ and

$$
\bar{d}\left(F_{i_{1} \ldots i_{q}} d x^{i_{1}} \wedge \cdots \wedge d x^{i_{q}}\right)=\left(\mathcal{D}\left(F_{i_{1} \ldots i_{q}}\right) \cdot d x\right) \wedge d x^{i_{1}} \wedge \cdots \wedge d x^{i_{q}},
$$

where $\mathcal{D}(F)=\partial_{x} F+\sum_{h>0} \partial_{Q_{(h)}} F\left(S_{(h+1)}+Q_{(h+1)}\right)$ is the operator of total derivation. The computation of the $S_{(r)}$, though complicated, is essentially a problem of linear algebra. This suggests the possibility of explicitly computing the characteristic cohomology of the Pfaffian systems considered in our discussion; details of this computation will appear in a subsequent publication.

\section{EXAMPLES}

4.1. Systems associated with a Cartan decomposition. Let $\mathfrak{g}=\mathfrak{g}_{0} \oplus \mathfrak{m}$ be a Cartan decomposition of a real semisimple Lie algebra $\mathfrak{g}$. We have the relations $\left[\mathfrak{g}_{0}, \mathfrak{g}_{0}\right] \subset \mathfrak{g}_{0},\left[\mathfrak{g}_{0}, \mathfrak{m}\right] \subset \mathfrak{m},[\mathfrak{m}, \mathfrak{m}] \subset \mathfrak{g}_{0}$, and $\mathfrak{g}_{0}$ and $\mathfrak{m}$ are orthogonal with respect to the Killing form $B$ of $\mathfrak{g}$. Moreover, the restrictions of $B$ to $\mathfrak{g}_{0}$ and $\mathfrak{m}$ are negative and positive definite, respectively.

\footnotetext{
${ }^{2}$ For a detailed description of the characteristic complex and of the corresponding cohomology theory we refer the reader to the article of Bryant and Griffiths [3].
} 
Let $\mathfrak{a} \subset \mathfrak{m}$ be a maximal ( $n$-dimensional) abelian subspace. The subspace $\mathfrak{m}$ decomposes as $\mathfrak{m}=\mathfrak{a} \oplus \mathfrak{b}$, where $\mathfrak{b}=\mathfrak{a}^{\perp}$ is the orthogonal complement with respect to $B$. Next, let

$$
\mathfrak{g}_{\mathfrak{a}}=\left\{X \in \mathfrak{g}_{0}:[X, \mathfrak{a}]=0\right\} .
$$

Then $\mathfrak{g}_{0}=\mathfrak{g}_{\mathfrak{a}} \oplus \mathfrak{p}$, where $\mathfrak{p}$ denotes the orthogonal complement of $\mathfrak{g}_{\mathfrak{a}}$ in $\mathfrak{g}_{0}$ with respect to the Killing form. Now, if $A \in \mathfrak{a}$ is a regular element, the mappings

$$
\left.\operatorname{ad}_{A}\right|_{\mathfrak{b}}: \mathfrak{b} \rightarrow \mathfrak{p},\left.\quad \operatorname{ad}_{A}\right|_{\mathfrak{p}}: \mathfrak{p} \rightarrow \mathfrak{b}
$$

define vector space isomorphisms between $\mathfrak{b}$ and $\mathfrak{p}$. Moreover, since $[\mathfrak{p}, \mathfrak{a}] \subset \mathfrak{b}$ and the map

$$
\mathfrak{p} \ni X \mapsto-\left.\operatorname{ad}_{X}\right|_{\mathfrak{a}} \in \operatorname{Hom}(\mathfrak{a}, \mathfrak{b})
$$

is injective, $\mathfrak{p}$ can be realized as a tableau, say $\mathbf{P}$, in $\operatorname{Hom}(\mathfrak{a}, \mathfrak{b})$. Similarly, as $[\mathfrak{b}, \mathfrak{a}] \subset \mathfrak{p}$ and the map $\left.\mathfrak{b} \ni B \mapsto \operatorname{ad}_{B}\right|_{\mathfrak{a}} \in \operatorname{Hom}(\mathfrak{a}, \mathfrak{p})$ is injective, $\mathfrak{b}$ can be realized as a tableau $\mathbf{A}$ in $\operatorname{Hom}(\mathfrak{a}, \mathfrak{p})$.

Now let $\Phi: \mathbf{A} \rightarrow \Lambda^{2}\left(\mathfrak{a}^{*}\right) \otimes \mathfrak{p}$ be the polynomial map defined by

$$
\left.\Phi\right|_{B}\left(A_{1}, A_{2}\right):=\left[\left[A_{1}, B\right],\left[A_{2}, B\right]\right] .
$$

A and $\Phi$ define the following system of partial differential equations for maps $F: U \subset \mathfrak{a} \rightarrow \mathfrak{b}, U$ open set of $\mathfrak{a}$,

$$
\left[A_{i}, \partial_{x^{j}} F\right]-\left[A_{j}, \partial_{x^{i}} F\right]=\left[\left[A_{i}, F\right],\left[A_{j}, F\right]\right], \quad 1 \leq i<j \leq n,
$$

where $\left(A_{1}, \ldots, A_{n}\right)$ is a basis of regular elements of $\mathfrak{a}$ and $\left(x^{1}, \ldots, x^{n}\right)$ denote the corresponding coordinates. This system in known in the literature as the $G / G_{0}$ system [1, 15] and, in a different formulation, as the the curved flat system [6, 17. We show that 4.1) fits into our general scheme by proving the following.

Proposition 4.1. The tableau $\mathbf{A}$ is involutive, $\Phi$ takes values in $B^{0,2}(\mathbf{A})$ and satisfies (3.1).

Proof. A direct computation shows that the characters of $\mathbf{A}$ are

$$
s_{1}^{\prime}=m-n=\operatorname{dim} \mathfrak{b}, \quad s_{2}^{\prime}=\cdots=s_{n}^{\prime}=0 .
$$

Next, observe that an element $F \in \operatorname{Hom}(\mathfrak{a}, \mathfrak{b})$ belongs to $\mathbf{A}^{(1)}$ if and only if $\left[F\left(A^{\prime}\right), A^{\prime \prime}\right]-\left[A^{\prime}, F\left(A^{\prime \prime}\right)\right]=0$, for all $A^{\prime}, A^{\prime \prime} \in \mathfrak{a}$. On the other hand, from Jacobi identity we get $\left[\operatorname{ad}_{X}\left(A^{\prime}\right), A^{\prime \prime}\right]-\left[A^{\prime}, \operatorname{ad}_{X}\left(A^{\prime \prime}\right)\right]=0$, for all $X \in \mathfrak{p}, A^{\prime}, A^{\prime \prime} \in \mathfrak{a}$. Therefore, the map $\left.\mathfrak{p} \ni X \rightarrow \operatorname{ad}_{X}\right|_{\mathfrak{a}} \in \mathbf{A}^{(1)}$ establishes an isomorphism between $\mathbf{A}^{(1)}$ and $\mathbf{P}$. This proves the involutiveness of $\mathbf{A}$ by counting dimensions.

For the second statement, note that

$$
2\left[\left[A_{1}, B\right],\left[A_{2}, B\right]\right]=\left[\left[B,\left[A_{1}, B\right]\right], A_{2}\right]-\left[\left[B,\left[A_{2}, B\right]\right], A_{1}\right],
$$

for $A_{1}, A_{2} \in \mathfrak{a}, B \in \mathfrak{b}$, and that $\left[B,\left[A_{1}, B\right]\right] \in \mathfrak{m}$. Next, define $S_{\left.\right|_{B}} \in C^{1,1}(\mathbf{A})$ by posing $S_{\left.\right|_{B}}(A):=[B,[A, B]]_{\left.\right|_{\mathfrak{b}}}$. We then have $\left.2 \Phi\right|_{B}\left(A_{1}, A_{2}\right)=\delta^{(1,1)}\left(S_{\left.\right|_{B}}\right)\left(A_{1}, A_{2}\right)$, for all $A_{1}, A_{2} \in \mathfrak{a}$. This proves that $\left.\Phi\right|_{B} \in B^{0,2}(\mathbf{A})$, for each $B \in \mathfrak{b}$. From the previous description of $\mathbf{A}^{(1)}$, we get

$$
\left.\Phi_{*}\right|_{(A, B)}\left(A_{1}+\operatorname{ad}_{X}\left(A_{1}\right)\right)\left(A_{2}, A_{3}\right)=\left[\left[A_{2},\left[X, A_{1}\right]\right],\left[A_{3}, B\right]\right]+\left[\left[A_{2}, B\right],\left[A_{3},\left[X, A_{1}\right]\right] .\right.
$$

Again using the Jacobi identity, we conclude that $\Phi$ satisfies (3.1). 
4.2. $1+1$ wave maps into Lie groups. A $1+1$ wave map is a smooth map $g: \mathbb{R}^{1,1} \rightarrow G$ into a Lie group $G$ that satisfies the differential equation

$$
\left(g^{-1} \partial_{x} g\right)_{y}+\left(g^{-1} \partial_{y} g\right)_{x}=0
$$

(see [14]). If $\theta=g^{-1} d g=A d x+B d y$, then $A, B: \mathbb{R}^{1,1} \rightarrow \mathfrak{g}$ satisfy the first order system

$$
\partial_{x} B=-[A, B], \quad \partial_{y} A=[A, B] .
$$

Conversely, if $A, B: \mathbb{R}^{1,1} \rightarrow \mathfrak{g}$ is a solution to (4.2), then $\theta=A d x+B d y$ satisfies the Maurer-Cartan equation and there exists a unique wave map $g: \mathbb{R}^{1,1} \rightarrow G$ such that $g^{-1} d g=\theta$ and $g(0,0)=e_{G}$ (the group identity).

Let $\mathfrak{a}=\mathbb{R}^{1,1}, \mathfrak{b}=\mathfrak{g} \oplus \mathfrak{g}$ and consider the tableau

$$
\mathbf{A}=\left\{\left(X_{2} d y, X_{1} d x\right): X_{1}, X_{2} \in \mathfrak{g}\right\} \subset \operatorname{Hom}(\mathfrak{a}, \mathfrak{b}),
$$

which we identify with $\mathfrak{b}$. Next, let

$$
\Phi:(A, B) \in \mathbf{A} \rightarrow-([A, B],[A, B])^{T} d x \wedge d y \in \Lambda^{2}\left(\mathfrak{a}^{*}\right) \otimes \mathfrak{b} .
$$

It is easily seen that (4.2) amounts to the system associated with the tableau $\mathbf{A}$ and the non-homogeneous term $\Phi$. Since there are two independent variables, $\Phi$ automatically satisfies (3.1). We need to prove that the tableau is 2-acyclic and that $\Phi$ takes values in $B^{0,2}(\mathbf{A})$. The first and second prolongation of $\mathbf{A} \simeq \mathfrak{b}$ are given, respectively, by

$$
\mathbf{A}^{(1)}=\left\{Q_{(1)}\left(\dot{X}_{1}, \dot{X}_{2}\right)=\left(\dot{X}_{2} d y, \dot{X}_{1} d x\right): \dot{X}_{1}, \dot{X}_{2} \in \mathfrak{g}\right\} \subset \Lambda^{1}\left(\mathfrak{a}^{*}\right) \otimes \mathbf{A}
$$

and

$\mathbf{A}^{(2)}=\left\{Q_{(2)} \mid Q_{(2)}\left(\ddot{X}_{1}, \ddot{X}_{2}\right)=\left(\ddot{X}_{2} d y \odot d y, \ddot{X}_{1} d x \odot d x\right): \ddot{X}_{1}, \ddot{X}_{2} \in \mathfrak{g}\right\} \subset S^{2}\left(\mathfrak{a}^{*}\right) \otimes \mathbf{A}$.

Therefore, $\operatorname{dim} \mathbf{A}^{(h)}=2 n$, for $h=0,1,2, s_{1}^{0}=n, s_{2}^{0}=n, s_{1}^{(1)}=2 n$ and $s_{2}^{(1)}=0$. We then conclude that the involutiveness index of $\mathbf{A}$ is $k=1$ and that the involutive characters are $s_{1}^{(1)}=2 n, s_{2}^{(1)}=0$. Since $\mathbf{A}^{(1)}$ is involutive, to prove that $\mathbf{A}$ is 2 acyclic we only need to prove that $H^{1,2}(\mathbf{A})=(0)$. In the case of two independent variables, this amounts to prove the surjectivity of $\delta^{2,1}$. For this, it is sufficient to observe that

$$
C^{2,1}(\mathbf{A})=\left\{\mu=\left(\mathcal{X}_{2} \otimes d y, \mathcal{X}_{1} \otimes d x\right): \mathcal{X}_{i}=X_{i 1} d x+X_{i 2} d y \in \Lambda^{1}\left(\mathfrak{a}^{*}\right) \otimes \mathfrak{g}\right\}
$$

and that $\delta^{(2,1)}(\mu)=\left(X_{21}, X_{12}\right) d x \wedge d y$. Finally, we show that $\Phi$ takes values in $B^{0,2}(\mathbf{A})$ by proving the vanishing of $H^{0,2}(\mathbf{A})$, which in turn amounts to proving that $\delta^{1,1}$ is onto. The claim follows by observing that

$$
C^{1,1}(\mathbf{A})=\left\{\sigma=\left(\mathcal{X}_{2}, \mathcal{X}_{1}\right): \mathcal{X}_{i}=X_{i 1} d x+X_{i 2} d y \in \Lambda^{1}\left(\mathfrak{a}^{*}\right) \otimes \mathfrak{g}\right\}
$$

and that $\delta^{1,1}(\sigma)=\left(X_{2,1},-X_{12}\right) d x \wedge d y$.

\section{The Cauchy problem}

5.1. Statement of the result. Retaining the notation of Sections 2 and 3 let $\mathcal{A}=\left(A_{1}, \ldots, A_{n}\right)$ be a basis of $\mathfrak{a}$ and let $\mathcal{Q}^{h}=\left(Q_{1}^{(h)}, \ldots, Q_{r_{h}}^{(h)}\right)$ be a basis of the prolongation $\mathbf{A}^{(h)}(h=0,1, \ldots, k)$, where $k$ is the least integer such that the prolongation $\mathbf{A}^{(k)}$ is involutive. Next, let $\mathcal{Q}^{k}=\left(Q_{[1], 1}^{(k)}, \ldots, Q_{[1], s_{1}}^{(k)}, \ldots, Q_{[\nu], 1}^{(k)}, \ldots, Q_{[\nu], s_{\nu}}^{(k)}\right)$ be a normal basis of $\mathbf{A}^{(k)}$ and suppose that its elements be in Guillemin's normal form with respect to $\mathcal{A}$ and $\mathcal{Q}^{k-1}$ (cf. Section 2.3). 
We call $\left(\mathcal{A}, \mathcal{Q}^{0}, \ldots, \mathcal{Q}^{k}\right)$ a regular basis of $M_{(k)}$. Let $\mathbf{A}^{(k)}=\mathbf{A}_{[1]}^{(k)} \oplus \cdots \oplus \mathbf{A}_{[\nu]}^{(k)}$, where $\mathbf{A}_{[p]}^{(k)}=\operatorname{span}\left(Q_{[p], 1}^{(k)}, \ldots, Q_{[p], s_{p}}^{(k)}\right), p=1, \ldots, \nu$. Consider the filtration

$$
\mathfrak{a}_{1}=\operatorname{span}\left(A_{1}\right) \subset \mathfrak{a}_{2}=\operatorname{span}\left(A_{1}, A_{2}\right) \subset \cdots \subset \mathfrak{a}_{\nu}=\operatorname{span}\left(A_{1}, \ldots, A_{\nu}\right) \subset \mathfrak{a} ;
$$

and denote by $\pi_{\rho}: \mathfrak{a} \rightarrow \mathfrak{a}_{\rho}$ the projection of $\mathfrak{a}$ on $\mathfrak{a}_{\rho}$ with respect to the decomposition $\mathfrak{a}=\mathfrak{a}_{\rho} \oplus \operatorname{span}\left(A_{\rho+1}, \ldots, A_{n}\right)$.

Definition 5.1. A set of Cauchy data consists of an ordered set

$$
\left(U, x_{0}, P_{(0)}, \ldots, P_{(k-1)}, P_{(k)}^{[1]}, \ldots, P_{(k)}^{[\nu]}\right),
$$

where $U \subset \mathfrak{a}$ is an open set of $x_{0} \in \mathfrak{a}, P_{(0)} \in \mathbf{A}^{(0)}, \ldots, P_{(k-1)} \in \mathbf{A}^{(k-1)}$ are constants, and $P_{(k)}^{[\rho]}: \pi_{\rho}(U) \subset \mathfrak{a}_{\rho} \rightarrow \mathbf{A}_{[\rho]}^{(k)}(\rho=1, \ldots, \nu)$ are analytic maps.

Theorem 5.2. Let $\mathbf{A} \subset \operatorname{Hom}(\mathfrak{a}, \mathfrak{b})$ be a 2-acyclic tableau, $k$ the least integer such that the prolongation $\mathbf{A}^{(k)}$ is involutive and $s_{1}^{(k)}, \ldots, s_{\nu}^{(k)}$ the corresponding principal characters. Let $\Phi: \mathfrak{a} \oplus \mathbf{A} \rightarrow \mathfrak{b} \otimes \Lambda^{2}\left(\mathfrak{a}^{*}\right) \subset B^{0,2}$ be an analytic function satisfying (3.1) and $\left(M_{(k)}, \mathfrak{I}_{(k)}, \Omega\right)$ the involutive prolongation of the differential system $(M, \mathfrak{I}, \Omega)$ defined by $\mathbf{A}$ and $\Phi$ (cf. Theorem [3.4). Let

$$
\left(U, x_{0}, P_{(0)}, \ldots, P_{(k-1)}, P_{(k)}^{[1]}, \ldots, P_{(k)}^{[\nu]}\right)
$$

be a set of analytic Cauchy data. Then there exist an open set $\widetilde{U} \subset U, x_{0} \in \widetilde{U}$, and an analytic map $Q=\left(Q_{(0)}, \ldots, Q_{(k)}\right): U \rightarrow \mathbf{A}^{[k]}$ such that:

(1) $\widetilde{U} \ni x \mapsto(x, Q(x)) \in M_{(k)}$ is a K-regular integral manifold of $\left(\Im_{(k)}, \Omega\right)$;

(2) $Q$ satisfies the initial conditions $Q_{(h)}\left(x_{0}\right)=P_{(h)}, h=0, \ldots, k-1$ and $Q_{(k)}^{[\rho]} \circ \pi_{\rho}=P_{(k)}^{[\rho]}$, for all $\rho=1, \ldots, \nu$

(3) $Q$ is unique, in the sense that any other analytic map with the same properties coincides with $Q$ in a neighborhood of $x_{0}$.

Remark 5.3. If the characteristic variety of $\mathbf{A}^{(k)}$ is hyperbolic (cf. [17), the result holds also in the case of $C^{\infty}$ data.

5.2. Proof of Theorem 5.2, The proof will be divided in several steps. Consider the vector bundles defined by

$$
J^{\perp}=\operatorname{Ker}\left(\beta_{(0)}, \ldots, \beta_{(k-1)}, d x^{1}, \ldots, d x^{n}\right) \subset I^{\perp}=\operatorname{Ker}\left(\beta_{(0)}, \ldots, \beta_{(k-1)}\right) \subset T M_{(k)} .
$$

Let $G_{h}(I, J) \rightarrow M_{(k)}$ be the Grassmann bundle of $h$-dimensional subspaces of $I^{\perp}$ transverse to $J^{\perp}$ and denote by $V_{h}(I, J) \subset G_{h}(I, J)$ the set of $h$-dimensional integral elements of $\left(\mathfrak{I}_{(k)}, \Omega\right)$. Let

$$
(P, \xi) \in M_{(k)} \times \mathfrak{a} \rightarrow V(\xi)_{P}=\xi+\sum_{h=1}^{k}\left(\left.S_{(h)}\right|_{P}+Q_{h}\right)(\xi)
$$

and consider the trivialization of $I^{\perp}$ given by

$$
(P, \xi, \mathcal{X}) \in M_{(k)} \times\left(\mathfrak{a} \oplus \mathbf{A}^{(k)}\right) \rightarrow V(\xi)_{P}+\mathcal{X} \in I^{\perp},
$$

for $P=\left(x, Q_{(0)}, \ldots, Q_{(k)}\right) \in M_{(k)}, \xi \in \mathfrak{a}, \mathcal{X} \in \mathbf{A}^{(k)}$, and the identifications

$$
G_{h}(I, J) \simeq M \times G_{h}\left(\mathfrak{a} \oplus \mathbf{A}^{(k)}, \mathbf{A}^{(k)}\right), \quad G_{h}\left(I^{\perp} / J^{\perp}\right) \simeq M \times G_{h}(\mathfrak{a}),
$$


where $G_{h}\left(\mathfrak{a} \oplus \mathbf{A}^{(k)}, \mathbf{A}^{(k)}\right)$ denotes the Grassmannian of $h$-dimensional subspaces in $\mathfrak{a} \oplus \mathbf{A}^{(k)}$ transverse to $\mathbf{A}^{(k)}$. Further, $G_{h}\left(\mathfrak{a} \oplus \mathbf{A}^{(k)}, \mathbf{A}^{(k)}\right)$ may be identified with the fiber bundle $\operatorname{Hom}\left(\mathcal{T}_{h}, \mathbf{A}^{(k)}\right)$ via the isomorphism

$\left(\mathfrak{a}_{h}, F\right) \in \operatorname{Hom}\left(\mathcal{T}_{h}, \mathbf{A}^{(k)}\right) \rightarrow \mathcal{A}_{h}\left(\mathfrak{a}_{h}, F\right):=\left\{\xi+F(\xi): \xi \in \mathfrak{a}_{h}\right\} \in G_{h}\left(\mathfrak{a} \oplus \mathbf{A}^{(k)}, \mathbf{A}^{(k)}\right)$, where $\mathcal{T}_{h} \rightarrow G_{h}(\mathfrak{a})$ denotes the tautological bundle. Therefore, we can think of $V_{h}(I, J)$ as an analytic subset of $M_{(k)} \times \operatorname{Hom}\left(\mathcal{T}_{h}, \mathbf{A}^{(k)}\right)$. Next, let $G_{h}^{\sharp}(\mathfrak{a}) \subset G_{h}(\mathfrak{a})$ be the Zariski open of $h$-dimensional generic subspaces with respect to the tableau $\mathbf{A}^{(k)}$ and let $V_{h}^{\sharp}(I, J) \subset V_{h}(I, J)$ the Zariski open of integral elements that project on $h$-dimensional generic subspaces.

Lemma 5.4. The projection $V_{h}^{\sharp}(I, J) \rightarrow G_{h}^{\sharp}(\mathfrak{a})$ is an (analytic) affine bundle, whose fibers have dimension $s_{1}+2 s_{2}+\cdots+(h-1) s_{h}+h\left(s_{h}+\cdots+s_{n}\right)$.

Proof. The relative tableau $\mathbf{A}^{(p)} \|_{\mathfrak{a}_{h}}:=\left\{\left.Q\right|_{\mathfrak{a}_{h}} \mid Q \in \mathbf{A}^{(p)}\right\}$ of a generic subspace $\mathfrak{a}_{h} \subset \mathfrak{a}$ has dimension $s_{1}+\cdots+s_{p}$. Therefore,

$$
\mathcal{A}_{h}^{(p)}=\left\{\left(\mathfrak{a}_{h}, Q_{\left.(p)\right|_{\mathfrak{a}_{h}}}\right): \mathfrak{a}_{h} \in G_{h}^{\sharp}(\mathfrak{a}), Q_{(p)} \in \mathbf{A}^{(p)}\right\} \rightarrow G_{h}^{\sharp}(\mathfrak{a})
$$

is a vector bundle of rank $s_{1}+\cdots+s_{p}$. The involutiveness of $\mathbf{A}^{(k)}$ implies that the morphism $\hat{\delta}: \operatorname{Hom}\left(\mathcal{T}_{h}^{\sharp}, \mathcal{A}_{h}^{(k)}\right) \rightarrow \Lambda^{2}\left(\mathcal{T}_{h}^{\sharp}\right) \otimes \mathcal{A}_{h}^{(k-1)}$ defined by

$$
\hat{\delta}(F)\left(\xi_{1}, \xi_{2}\right)=F\left(\xi_{1}\right)\left(\xi_{2}\right)-F\left(\xi_{2}\right)\left(\xi_{1}\right),
$$

for $F \in \operatorname{Hom}\left(\mathfrak{a}_{h}, \mathcal{A}_{h}^{(k)} \mid \mathfrak{a}_{h}\right)$, and $\xi_{1}, \xi_{2} \in \mathfrak{a}_{h}$, has constant rank.

The kernel of $\hat{\delta}$, which we denote by $\left(\mathcal{A}_{h}^{(k+1)}\right)^{(1)}$, is a subbundle, whose fiber above $\mathfrak{a}_{h}$ is the first prolongation of $\mathbf{A}_{\|_{a_{h}}}^{(k)}$. To compute the dimension of the fibers it suffices to observe that $\mathbf{A}_{\|_{\mathfrak{a}_{h}}}^{(k)}$ is involutive, from which it follows that $\operatorname{dim}\left(\mathbf{A}_{\|_{\mathfrak{a}_{h}}}^{(k)}\right)^{(1)}=s_{1}+2 s_{2}+\cdots+h s_{h}$.

Let $\rho: \operatorname{Hom}\left(\mathcal{T}_{h}^{\sharp}, \mathbf{A}^{(k)}\right) \rightarrow \operatorname{Hom}\left(\mathcal{T}_{h}^{\sharp}, \mathcal{A}_{h}^{(k)}\right)$ be the restriction morphism defined by

$$
\rho(F)(\xi)=\left.F(\xi)\right|_{\mathfrak{a}_{h}}, \text { for } F \in \operatorname{Hom}\left(\mathfrak{a}_{h}, \mathbf{A}^{(k)}\right), \xi \in \mathfrak{a}_{h}, \mathfrak{a}_{h} \in G_{h}^{\sharp}(\mathfrak{a}) .
$$

Let $\Psi: \operatorname{Hom}\left(\mathcal{T}_{h}^{\sharp}, \mathbf{A}^{(k)}\right) \rightarrow \operatorname{Hom}\left(\mathcal{T}_{h}^{\sharp}, \mathcal{A}_{h}^{(k)}\right) /\left(\mathcal{A}_{h}^{(k)}\right)^{(1)}$ be the composition of $\rho$ with the projection on the quotient and let $\hat{S}_{(k+1)}: M_{(k)} \times G_{h}(\mathfrak{a}) \rightarrow M_{(k)} \times \operatorname{Hom}\left(\mathcal{T}_{h}, \mathbf{A}^{(k)}\right)$ be the section defined by $\left(P, \mathfrak{a}_{h}\right) \rightarrow\left(P, S_{(k+1)} \mid P \|_{\mathfrak{a}_{h}}\right)$. The proof of the lemma follows by observing that

$$
V^{\sharp}(I, J)=\left\{(P, F) \in M_{(k)} \times \operatorname{Hom}\left(\mathcal{T}_{h}^{\sharp}, \mathbf{A}^{(k)}\right): \Psi\left(F-\left.\hat{S}_{(k+1)}\right|_{P}\right)=0\right\}
$$

and that $\operatorname{rank}(\operatorname{Ker}(\Psi))=s_{1}+2 s_{2}+\cdots+(h-1) s_{h-1}+h\left(s_{h}+\cdots+s_{n}\right)$.

Lemma 5.5. The generic integral flags are $K$-regular.

Proof. For any $P \in M_{(k)}$ and for any $\mathcal{A}_{h} \in G_{h}^{\sharp}\left(\mathfrak{a} \oplus \mathbf{A}^{(k)}, \mathbf{A}^{(k)}\right)$ consider the polar space

$\mathcal{H}\left(P, \mathcal{A}_{h}\right):=\left\{\xi_{1}+\mathcal{X}_{1} \in \mathfrak{a} \oplus \mathbf{A}^{(k)}:\left(\pi_{(k)} \dot{\wedge} \eta\right)\left(\xi_{1}+\mathcal{X}_{1}, \xi+\mathcal{X}\right)=0\right.$, for all $\left.\xi+\mathcal{X} \in \mathcal{A}_{h}\right\}$

Since the projection of $\mathcal{A}_{h}$ on $\mathfrak{a}$ is a generic subspace, we have that

$$
\operatorname{dim}\left(\mathcal{H}\left(P, \mathcal{A}_{h}\right)\right)=n+\left(s_{h+1}+\cdots+s_{\nu}\right) .
$$


If $\mathcal{A}_{0}=(0) \subset \mathcal{A}_{1} \subset \cdots \subset \mathcal{A}_{n} \subset \overline{\mathfrak{a}} \oplus \mathbf{A}^{(k+1)}$ is an integral flag in $P \in M_{(k)}$ and if the $\mathcal{A}_{h}$ are generic, then

$$
\operatorname{dim} \mathcal{H}\left(P, \mathcal{A}_{h}\right)=n+\left(s_{h+1}+\cdots+s_{\nu}\right)
$$

for $h=0, \ldots, n$. From this identities and the preceding lemma it follows that $\left(\mathcal{A}_{0}, \ldots, \mathcal{A}_{n}\right)$ is an integral flag which is $K$-regular.

Lemma 5.6. let $\left(\mathcal{A}, \mathcal{Q}^{0}, \ldots, \mathcal{Q}^{k}\right)$ be a regular basis and $\mathcal{A}_{h+1} \subset \mathfrak{a} \oplus \mathbf{A}_{[h+1]}^{(k)} \oplus \cdots \oplus$ $\mathbf{A}_{[\nu]}^{(k)}$ an $(h+1)$-dimensional subspace. Assume that $\mathcal{A}_{h+1}$ projects onto $\mathfrak{a}_{h+1} \subset \mathfrak{a}$ and set

$$
\Sigma\left(\mathcal{A}_{h+1}\right):=\mathbf{A}^{(0)} \oplus \cdots \oplus \mathbf{A}^{(k-1)} \oplus\left(\mathcal{A}_{h+1} \oplus \mathbf{A}_{[1]}^{(k)} \oplus \cdots \oplus \mathbf{A}_{[h]}^{(k)}\right) .
$$

If $\mathcal{A}_{h}$ is an h-dimensional integral element at $P \in M_{(k)}$ contained in $\Sigma\left(\mathcal{A}_{h+1}\right)$ and if $\mathcal{A}_{h}$ projects onto $\mathfrak{a}_{h} \subset \mathfrak{a}_{h+1}$, then $\operatorname{dim}\left(\mathcal{H}\left(P, \mathcal{A}_{h}\right) \cap \Sigma\left(\mathcal{A}_{h+1}\right)\right)=h+1$.

Proof. First observe that $\mathcal{H}\left(P, \mathcal{A}_{h}\right) \cap \Sigma\left(\mathcal{A}_{h+1}\right)=\mathcal{H}\left(P, \mathcal{A}_{h}\right) \cap\left(\Sigma\left(\mathcal{A}_{h+1}\right) \cap I^{\perp}{ }_{{ }_{P}}\right)$, and that the elements of $\left.\Sigma\left(\mathcal{A}_{h+1}\right) \cap I^{\perp}\right|_{P}$ are of the form $V_{P}(\xi)+\mathcal{X}(\xi)+\mathcal{S}$, where $\xi \in \mathfrak{a}_{h+1}, \mathcal{X} \in \operatorname{Hom}\left(\mathfrak{a}_{h+1}, \mathbf{A}_{[h+1]}^{(k)} \oplus \cdots \oplus \mathbf{A}_{[\nu]}^{(k)}\right)$ and $\mathcal{S} \in \mathbf{A}_{[1]}^{(k)} \oplus \cdots \oplus \mathbf{A}_{[h]}^{(k)}$. Next, the elements of $\mathcal{A}_{h}$ are of the form $V_{P}(\xi)+\mathcal{X}(\xi)+\mathcal{Y}(\xi)$, where $\xi \in \mathfrak{a}_{h}$ and $\mathcal{Y} \in$ $\operatorname{Hom}\left(\mathfrak{a}_{h}, \mathbf{A}_{[1]}^{(k)} \oplus \cdots \oplus \mathbf{A}_{[h]}^{(k)}\right)$. If $\mathfrak{r} \subset \mathfrak{a}_{h+1}$ is a 1-space such that $\mathfrak{a}_{h+1}=\mathfrak{r} \oplus \mathfrak{a}_{h}$, the elements of $\Sigma\left(\mathcal{A}_{h+1}\right) \cap I^{\perp}{ }_{P}$ are the form

$$
Y\left(\xi, \xi^{\prime}, \mathcal{S}\right)=\left(V_{P}(\xi)+\mathcal{X}(\xi)+\mathcal{Y}(\chi)\right)+\left(V_{P}\left(\xi^{\prime}\right)+\mathcal{X}\left(\xi^{\prime}\right)\right)+\mathcal{S},
$$

where $\xi \in \mathfrak{a}_{h}, \xi^{\prime} \in \mathfrak{r}$ and $\mathcal{S} \in \mathbf{A}_{[1]}^{(k+1)} \oplus \cdots \oplus \mathbf{A}_{[h]}^{(k+1)}$.

If we impose that $Y\left(\xi, \xi^{\prime}, \mathcal{S}\right)$ satisfy the polar equations, then (using the fact that the elements of the basis $\mathcal{Q}^{k}$ are in normal form) we find that $\mathcal{S}$ is determined by $\xi$ and then $\operatorname{dim}\left(\mathcal{H}\left(P, \mathcal{A}_{h}\right) \cap \Sigma\left(\mathcal{A}_{h+1}\right)\right)=h+1$.

Let $\left(x, Q_{(0)}, \ldots, Q_{(k)}, Q_{(k)}^{[1]}, \ldots, Q_{(k)}^{[\nu]}\right)$ be the coordinates with respect to the regular basis. Consider a set $\left(U, x_{0}, P_{(0)}, \ldots, P_{(k-1)}, P_{(k)}^{[1]}, \ldots, P_{(k)}^{[\nu]}\right)$ of analytic Cauchy data and the filtration of submanifolds

$$
\mathcal{N}_{0} \subset \mathcal{N}_{1} \subset \cdots \subset \mathcal{N}_{\nu} \subset \mathcal{N}_{\nu+1} \subset \cdots \subset \mathcal{N}_{n}=M
$$

defined as follows:

- $\mathcal{N}_{0}$ is the point of coordinates $x=x_{0}, Q^{(h)}=P_{(h)}, Q_{(k)}^{[p]}=P_{(k)}^{[p]}\left(x_{0}^{1}, \ldots, x_{0}^{p}\right)$, for $h=1, \ldots, k-1$ and $p=1, \ldots, \nu$;

- for $r=1, \ldots, \nu, \mathcal{N}_{r}$ is defined by the equations $x^{r+1}=x_{0}^{r+1}, \ldots, x^{n}=x_{0}^{n}$, $Q_{(k)}^{[r+p]}=P_{(k)}^{[r+p]}\left(x^{1}, \ldots, x^{r}, x_{0}^{r+1}, \ldots, x_{0}^{\nu}\right)$, for $p=1, \ldots, \nu-r$

- for $r=\nu+1, \ldots, n, \mathcal{N}_{r}$ is defined by $x^{r+1}=x_{0}^{r+1}, \ldots, x^{n}=x_{0}^{n}$.

From the preceding lemma it follows that if $\mathcal{A}_{h} \subset T_{P}\left(\mathcal{N}_{h+1}\right)$ is an $h$-dimensional integral element, then $\operatorname{dim}\left(\mathcal{H}\left(P, \mathcal{A}_{h}\right) \cap T_{P}\left(\mathcal{N}_{h+1}\right)\right)=h+1$. By applying the CartanKähler theorem we prove the existence of a unique filtration of $K$-regular integral submanifolds $P_{1} \subset \cdots \subset P_{\nu} \subset P_{\nu+1} \subset \cdots \subset P_{n}$, $\operatorname{dim} P_{h}=h$, such that $\mathcal{N}_{h-1} \subset$ $P_{h} \subset \mathcal{N}_{h}$, for $h=1, \ldots, n$.

Let $\pi_{1}: P_{n} \rightarrow \mathfrak{a}$ and $\pi_{2}: P_{n} \rightarrow \mathbf{A}^{[k]}$ be the restrictions of the respective projections of $M_{(k)}$. Since $\pi_{1}$ has maximal rank we may assume that the image be an open neighborhood $\widetilde{U}$ of $x_{0}$ and that $\pi_{1}$ be invertible. Thus $Q=\pi_{2} \circ\left(\pi_{1}\right)^{-1}$ : 
$\widetilde{U} \rightarrow \mathbf{A}^{[k]}$ satisfies the requested properties. The uniqueness of $Q$ is a consequence of the uniqueness of the $K$-regular integral submanifolds $P_{1} \subset \cdots \subset P_{n}$ such that $\mathcal{N}_{h-1} \subset P_{h} \subset \mathcal{N}_{h}, h=1, \ldots, n$. This proves the theorem.

\section{REFERENCES}

[1] M. Brück, X. Du, J. Park, C.-L. Terng, Submanifold geometry of real Grassmannian systems, Mem. Amer. Math. Soc. 155 (2002), no. 735, 1-95.

[2] R. L. Bryant, S.-S. Chern, R. B. Gardner, H. L. Goldschmidt, P. A. Griffiths, Exterior differential systems, Mathematical Sciences Research Institute Publications, 18, SpringerVerlag, New York, 1991.

[3] R. L. Bryant, P. A. Griffiths, Characteristic cohomology of differential systems, I: General theory, J. Amer. Math. Soc. 8 (1995), 507-596.

[4] B. Dai, C.-L. Terng, Periodic and homoclinic solutions of the modified $2+1$ chiral model, J. Math. Phys. 46 (2005), no. 6, 2706-2719.

[5] B. Dai, C.-L. Terng, Backlund transformations, Ward solitons, and unitons, 2004. Preprint available as math.DG/0405363 on the arXiv.

[6] D. Ferus, F. Pedit, Curved flats in symmetric spaces, Manuscripta Math. 91 (1996), 445-454.

[7] _ Isometric immersions of space forms and soliton theory, Math. Ann. 305 (1996), 329-342.

[8] C. H. Gu, On the Cauchy problem for harmonic maps defined on two-dimensional Minkowski space, Comm. Pure Appl. Math. 33 (1980), 727-737.

[9] V. Guillemin, Some algebraic results concerning the characteristics of overdetermined partial differential equations, Amer. J. Math. 90 (1968), 270-284.

[10] E. Musso, L. Nicolodi, Tableaux over Lie algebras, integrable systems and classical surface theory, Comm. Anal. Geom. (to appear). Preprint available as math.DG/0412169 on the arXiv.

[11] - On the Cauchy problem for the integrable system of Lie minimal surfaces, J. Math. Phys. 46 (2005), no. 11, 3509-3523.

[12] J. Shatah, W. Strauss, Breathers as homoclinic geometric wave maps, Phys. D 99 (1996), $113-133$.

[13] C.-L. Terng, Soliton equations and differential geometry, J. Differential Geom. 45 (1997), 407-445.

[14] C.-L. Terng, K. Uhlenbeck, $1+1$ wave maps into symmetric spaces. Comm. Anal. Geom. 12 (2004), 345-388.

[15] C.-L. Terng, E. Wang, Curved flats, exterior differential systems and conservation laws; Complex, Contact and Symmetric Manifolds. in honor of L. Vanhecke, 235-254, Progress in Mathematics, Vol. 234, O. Kowalski; E. Musso; D. Perrone (Eds.), Birkhäuser, 2005.

[16] K. Uhlenbeck, Harmonic maps into Lie groups: classical solutions of the chiral model, $J$. Differential Geom. 30 (1989), 1-50.

[17] D. Yang, Involutive hyperbolic differential systems, Mem. Amer. Math. Soc. 68 (1987), no. 370, 1-93.

(E. Musso) Dipartimento di Matematica Pura ed Applicata, Università degli Studi di L'Aquila, Via Vetoio, I-67010 Coppito (L'Aquila), Italy

E-mail address: musso@univaq.it

(L. Nicolodi) Dipartimento di Matematica, Università degli Studi di Parma, Parco Area delle Scienze 53/A, I-43100 Parma, Italy

E-mail address: lorenzo.nicolodi@unipr.it 